\title{
Patient Knowledge Compared With National Guidelines for Diabetes Care
}

\author{
SUSAN L. TEZA, RN, CDE \\ WAYNE K. DAVIS, PhD \\ ROLAND G. HISS, MD \\ Michigan Diabetes Research and Training Center \\ The University of Michigan Medical School \\ Ann Arbor, Michigan
}

\begin{abstract}
Data collected on a randomly selected group of 428 patients with insulin-dependent and noninsulin-dependent diabetes from 61 physician practices in eight Michigan communities were compared with national standards for diabetes patient knowledge. Comparisons were performed using a standardized Diabetes Knowledge Test (DKT) and selected items from the Diabetes Education Profile (DEP). Patient performance on these instruments was compared with corresponding items in the Ambulatory Care Facilities section of the Guidelines for Diabetes Care published by the American Diabetes Association and the American Association of Diabetes Educators. In general, insulin-dependent persons scored higher than noninsulin-dependent persons. Those taking insulin (whether insulin-dependent or not) scored higher than noninsulin-dependent persons whose regimen did not include insulin. The findings emphasize the need to subdivide any analysis of clinical diabetes or diabetes education into groups based on insulin use or nonuse.
\end{abstract}

The American Diabetes Association and the American Association of Diabetes Educators jointly published Guidelines for Diabetes Care in 1981.' One section of this document described suidelines for diabetes care in ambulatory care facilities. This section was divided into ten categories (called elements) as depicted in Table 1.

The guidelines for ambulatory care included patient knowledge and behavior as one end point of care and set detailed and rigorous standards for desired patient knowledge in nine of the ten elements. An evaluation of a large population of randomly selected diabetic patients has afforded an opportunity to compare these national standards for patient knowledge of diabetes with actual patient knowledge in the community setting. This comparison may serve as a needs assessment for the planning of diabetes education programs, and may have implications for the conduct of other aspects of diabetes care as well.

\section{Methods}

As part of the outreach activities of the Michigan Diabetes Research and Training Center (MDRTC), primary care physicians from four large and four small Michigan communities were randomly selected and seven patients with diabetes were randomly selected from each of their practices، In all, 428 patients from 61 practices in eight communities were evaluated by project staff. The medical and educational histories were obtained from each patient and blood samples taken for analysis of glucose and glycosylated hemoglobin $\left(\mathrm{HbA}_{1}\right)$. The patients were subdivided into three clinical groups: (1) insulin-dependent diabetes, (2) noninsulin-dependent diabetes managed with insulin, and (3) noninsulin-dependent diabetes not managed with insulin. Insulin-dependent patients were identified as those with the diagnosis of diabetes prior to age 30, continuous use of insulin since diagnosis, and body weight less than $120 \%$ of ideal. All other patients were con-

This work was supported by grant \#P60AM20572 from the National Institute of Arthritis, Diabetes, Digestive and Kidney Diseases, National Institutes of Health.

Reprint requests to Roland G. Hiss, MD, G1205 Towsley Center, Box 0201. The University of Michigan Medical Center, Ann Arbor. MI 48109-0201. 
Table 1. Major Elements of Guidelines for Diabetes Care in Ambulatory Care Facilities.*

I. Diagnosis Is Established

II. Nutrition Care Plan Is Designed

III. Individual Däily Physical Activity Plan Is Designed

IV. Insulin

V. Oral Hypoglycemic Agents

VI. Urine Testing ${ }^{+}$

VII. Hypoglycemia

VIII. Ketoacidosis

IX. Lower Extremities

$\mathrm{X}$. Periodic Evaluation

*From Guidelines for Diabetes Care. ${ }^{1}$

tThe 1981 guidelines did not utilize blood glucose monitoring.

sidered to have Type II diabetes, and insulin usage was determined by patient interview. (It is recognized that there may be as much as a $10 \%$ error in either direction in the definition of Type I and Type II diabetes using these criteria.) No attempt was made to confirm the diagnosis of diabetes as established by the attending physician as the purpose of the study was to evaluate the status of patients considered to have diabetes and being actively followed for this condition in a community setting. Most of the data collected on the patients were supportive of this diagnosis, however. A standardized diabetes knowledge test (DKT) and Diabetes Education Profile (DEP), both developed at the MDRTC, ${ }^{2,3}$ were administered to all patients.
Patient performance on the DKT and DEP* was assessed and compared with the corresponding items listed in the Ambulatory Care Facilities section of the ADA/AADE guidelines. The guidelines list ten elements (categories) of diabetes care and specify the desired level of patient knowledge that should result from this care for nine of the ten elements. Of the ten elements, this study provided comparison data for eight. (Guideline Element I, which relates to the diagnosis of diabetes, was not verified in our study; and the guidelines did not specify a patient knowledge component for this element. There were insufficient data in the DKT and DEP to measure Element V, Oral Hypoglycemia Agents.) In nine of the ten elements, the guidelines called for the patient and/or responsible person to give one or more particular response(s) or perform one or more particular skill(s) applicable to that element. We have termed these responses and skills patient knowledge items. Patient data from the DKT and DEP were available for 18 of the 51 patient knowledge items listed in the guidelines. The number of patient knowledge items that could be assessed for each element ranged from one to five. A sample of the standards from the guidelines is provided in Fig 1 to show the relationship of an element of diabetes care and its associated patient knowledge item(s).

The data were analyzed by combining the Diabetes Knowledge Test and Diabetes Education Profile questions pertaining to each patient knowledge item into one composite value for each clinical patient group. This was done by determining the percent of patients in each clinical group who answered a DKT or DEP question correctly and computing the arithmetic mean of these percents for all questions pertaining to a given patient knowledge item. The composite value so deter-

*The items used to make these assessments are available from the authors.

\section{Individual Daily Physical Activity $\longleftarrow$ Diabetes Care Plan Is Designed

Patient and/or responsible person

states and/or demonstrates:

1. Effects of activity on blood glucose level.

2. Relationship of activity to general health and well-being.

3. Need for daily physical activity.

4. Appropriate action to compensate for significantly increased or decreased physical activity.

5. Interrelationship of activity, time of meals, and hypoglycemic medication on blood glucose level.

Fig 1. Sample standard from Guidelines for Ambulatory Care Facilities section. This depicts Element III, Individual Daily Physical Activity Is Designed, and the five patient knowledge items listed for it. The items in italics represent those for which the assessment instruments of this study provided adequate data to determine community patient knowledge. 
mined was called an "average patient score" for that item. For each element, the average patient scores on the items subsumed by that element were similarly computed.

\section{Results}

A total of 428 patients from the practices of 61 primary care physicians were studied. There were 172 men and 256 women with an age range of 16 to 85 years (children had been deliberately excluded from the study). A profile of the 428 patients is shown in Table 2.

Table 3 displays the average patient scores on the knowledge assessments associated with the eight diabetes care elements for which comparison data were available.

Table 2. Patient Population Profile $(\mathrm{N}=428)$

Noninsulin- Noninsulin-

Insulin- Dependent Dependent Not

Dependent Using Insulin Using Insulin

\begin{tabular}{|c|c|c|c|}
\hline $\begin{array}{c}\text { Number of } \\
\text { patients }\end{array}$ & $\begin{array}{c}56 \\
\text { (39\% female) }\end{array}$ & $\begin{array}{c}191 \\
(65 \% \text { female })\end{array}$ & $\begin{array}{c}181 \\
\text { (61\% female) }\end{array}$ \\
\hline $\begin{array}{l}\text { Mean age and } \\
\text { range }\end{array}$ & $35(16-68)$ & $59(24-84)$ & $62(31-86)$ \\
\hline $\begin{array}{l}\text { Average duration } \\
\text { of diabetes } \\
\text { (years) }\end{array}$ & 19.6 & 13.3 & 7.9 \\
\hline $\begin{array}{l}\text { Formal diabetes } \\
\text { education ever }\end{array}$ & $44(78 \%)$ & $159(83 \%)$ & $96(53 \%)$ \\
\hline $\begin{array}{l}\text { Years since last } \\
\text { formal diabetes } \\
\text { education }\end{array}$ & 4.6 & 3.8 & 4.1 \\
\hline $\begin{array}{l}\text { Average } \mathrm{HbA}_{1} \\
(\%)^{*}\end{array}$ & 10.9 & 10.3 & 8.7 \\
\hline
\end{tabular}

Insulin-dependent patients $(n=56)$ had an overall score of $71 \%$; insulin-using noninsulin-dependent patients $(n=191)$ had an overall score of $57 \%$; the third clinical group, noninsulin-dependent patients not using insulin $(n=181)$, had an overall score of $52 \%$. In general, patients with insulindependent diabetes performed better on each of the eight categories than did those with noninsulin-dependent diabetes managed with insulin. The latter group, in turn, generally performed better than the group of noninsulin-dependent patients managed without insulin.

Table 4 lists the average patient scores of each clinical group for the 18 patient knowledge items. Of the 18 items examined, insulin-dependent patients scored $75 \%$ or better on 11 items; noninsulin-dependent patients using insulin attained a score of $75 \%$ or better on 4 items; and noninsulindependent patients not using insulin only met the $75 \%$ level on 1 item (6\%). No group achieved a score of $75 \%$ or better on all 18 items.

Some areas showed considerable deficiency, and it is likely that these weaknesses adversely affect overall outcome of diabetes care. Under Element II, Nutrition Care Plan Is Designed, the guidelines recommendation specifies that the patient (and/or responsible person) states and/or demonstrates the "Reason for following meal plan on a daily basis," "The daily meal plan," and "Maintenance of oral intake during periods of illness." Scores from all three clinical groups showed low results on these patient knowledge items. Many patients were unable to state why they were given a particular meal plan; they could not recognize examples of foods from the various food groups; and they were unable to state how they would maintain oral intake during periods of illness. The only item to which all three groups responded well was "Relationship of increased or decreased food intake to blood glucose level."

In Element VII, Hypoglycemia, insulin-dependent patients scored consistently higher on each of its knowledge items than did noninsulin-dependent patients. This patient group was more cognizant of the prevention. causes, recogni-

Table 3. Composite Scores on Patient Knowledge Assessments Associated With Diabetes Care Elements*

\section{Diabetes Care Element}

II. Nutrition Care Plan Is Designed

III. Individual Daily Physical Activity Plān Is Designed

IV. Insulin

VI. Urine Testing

VII. Hypoglycemia

VIII. Ketoacidosis

IX. Lower Extremities

X. Periodic Evaluation

\section{Insulin-Dependent Patients \\ $(\mathrm{n}=56)$}

$71 \%$

69

65

81

84

88

38

75

71

\author{
Noninsulin-Dependent \\ Patients Managed \\ With Insulin \\ $(n=191)$
}

$62 \%$

\section{0}

45

65

69

80

29

58

57

\section{Noninsulin-Dependent Patients Managed Without Insulin $(n=181)$}

$$
57 \%
$$

63

59

74

38

51

52 
Table 4. Average Patient Scores (Percent)

\section{Patient Knowledge Items}

1. Relationship of increased or decreased food intake to blood glucose level (II-1)*

2. Reason for following meal plan on a daily basis (II-3)

3. The daily meal plan (II-4)

4. Maintenance of oral intake during periods of illness (II-7)

5. Effects of activity on blood glucose level (III-1)

6. Relationship of activity to general health and well-being (III-2)

7. Interrelationship of activity, time of meals, and hypoglycemic medication on blood glucose level (III-5)

8. Injection sites and rotation pattern (IV-5)

9. Appropriate action for adjustment of daily insulin dose (IV-8)

10. Alteration of insulin dose(s) and meal plan according to urine test results (VI-6)

11. Prevention of hypoglycemia (VII-2)

12. Causes of hypoglycemia (VII-3)

13. Recognition of hypoglycemia (VII-4)

14. Treatment of hypoglycemia (VII-5)

15. Signs and symptoms of impending ketoacidosis (VIII-1)

16. Major causes of ketoacidosis (VIII-2)

17. Care of minor injuries to feet and lower extremities (IX-5)

18. Sick day rules $(X-2)$

\section{Insulin-Dependent \\ Patients \\ $(\mathbf{n}=56)$}

$97 \%$

68

64

54

78

54

74

54

76

81

79

85

76

95

85

91

38

75

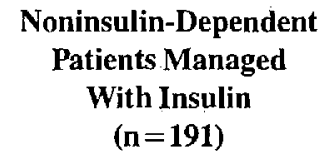

$82 \%$

64

58

43

50

53

48

46

48

15

42

48

65

60

65

71

79

76

83

29

58

60

50

32

40

(1)

57

64

72

45

74

73

38

51
Noninsulin-Dependent

Patients Managed

Without Insulin

$(\mathbf{n}=\mathbf{1 8 1})$

\section{$85 \%$}

*Indicates Diabetes Care Element and Patient Knowledge Item from Guidelines for Diabetes Care. ${ }^{1}$

tion, and treatment of hypoglycemia. The scores dropped stepwise in the other two clinical groups, presumably as a result of less personal experience with hypoglycemia. Scores of all three clinical groups were considerably higher on items selected from Element VIII, Ketoacidosis. Insulindependent and noninsulin-dependent patients using insulin scored above $75 \%$ on items that asked the "Signs and symptoms of impending ketoacidosis" and the "Major causes of ketoacidosis." In Element IX, Lower Extremities, all three patient groups showed poor results-38\%, 29\%, and $38 \%$, respectively. The implications of these low scores in both the clinical management and educational aspects of the care of all persons with diabetes are evident.

\section{Discussion}

The patients evaluated in this study were randomly chosen from the practices of primary care physicians in four large and four small communities, and the physicians themselves were randomly selected from among their colleagues in each community. To our knowledge this is the only study of a large randomly selected group of diabetes patients who were both selected from and studied in their community settings.

This analysis was performed to determine how a "typical diabetic patient" would do when his/her diabetes knowledge and skills were compared with a national standard. The standard used was the Guidelines for Diabetes Care developed and jointly published by the American Diabetes Association and the American Association of Diabetes Educators in 1981. Although there has been some evolution of standards since 1981, particularly in the area of self-monitoring of blood glucose, the Guidelines still reflect the majority of currently held standards and are the last major statement of standards to be published.

The chief purpose of the study was to alert health profes- 
sionals to areas of weakness in patient understanding (and possibly, therefore, of management) of the diabetic regimen. This information should be useful to physicians in the selection of various aspects of the management program of patients and to diabetes educators in their planning of diabetes education. The findings, however, apply to whole populations or categories of diabetic patients and do not directly apply to individual patients. In general, persons with insulindependent diabetes attained higher scores in most categories than did noninsulin-dependent persons. The difference between the three clinical groups analyzed in this study (insulin-dependent, noninsulin-dependent using insulin, and noninsulin-dependent not using insulin) serves to underline the importance of subdividing an analysis of clinical diabetes or diabetes education into groups based on insulin need and use.

The explanation for the better performance on a standardized knowledge test of insulin-taking patients as compared with noninsulin-taking patients is complex. A greater percentage of insulin-taking patients had received formal diabetes education than had noninsulin-taking patients (Table 2), but the elapsed time since this education was the same for all groups (approximately four years). History of formal diabetes education and current insulin use were both positively related to patient scores on a standardized diabetes knowledge test. Duration of diabetes was associated with improved scores only for the subset of patients who had never had formal diabetes education. Age was negatively correlated with test scores. ${ }^{4}$ The important point is, however, that regardless of reason, type of diabetes and current insulin use are often associated with differences in patient knowledge about the major principles of diabetes and diabetes care. It is important for diabetes educators to be aware of these differences when planning programs "for diabetes patients" or in the preparation of diabetes patient education instructional materials. The target audience of diabetes patient educational activities is quite heterogeneous and programs developed "for diabetes patients" should be developed with these differences in mind.

We recognize that patients not using insulin do not have a need to know insulin-related mechanics and insulinproduced effects. Their knowledge deficit in these areas is understandable and inconsequential. Their knowledge deficits in other aspects of diabetes care-diet, exercise, health maintenance, and foot care, for instance-is of concern, however. It would appear that the experience of using insulin is associated with an indirect learning process about multiple aspects of diabetes that patients not using insulin don't receive. Some of the "educational disadvantage" of diabetic patients for whom insulin has not been prescribed may be related to the attitude expressed by many health professionals (and patients) that this form of the disorder is mild and not deserving of much attention. This is the attitude characterized by the common descriptive phrase "touch of sugar" used by health professionals to reassure (inappropriately) their Type II patients.

It is apparent that the level of patient understanding of the diabetic regimen and the level of self-monitoring reported in the sampled communities fall short of the ideal described in the 1981 guidelines. Until the results of this study were reported to them, the practitioners in these communities were largely unaware of the level of patient understanding and adherence to the guidelines. The results of this study provide sobering data regarding the lack of guideline achievement. These results are also similar to widespread and substantial knowledge deficits about diabetes among diabetic patients and their families that have previously been reported in earlier studies. ${ }^{5-8}$ The current study shows, unfortunately, that the recent emphasis on diabetes patient education nationally has not as yet had universal effect at the community level. Our study was repeated in 1985 , when 261 of the original 428 patients $(61 \%)$ were relocated and agreed to be retested with the same instruments. Essentially no change in patient knowledge, analyzed in aggregate, had occurred in the fouryear interval. ${ }^{+}$

An important limitation of the current study should be noted. Since the purpose of our original study had not been to determine the level of guideline achievement, the questions included in the Diabetes Knowledge Test and the Diabetes Educational Profile did not measure everything included in the guidelines. However, in selecting questions to measure guideline attainment, the investigators were conservative and only DKT and DEP questions that exactly matched guideline items were used. If a future study of current practices is planned, instruments specific to the guidelines should be developed and validated for this use.

The authors thank George Hess, BS, MA, Program Manager in Postgraduate Medicine and Health Professions Education, for his assistance in analyzing the data collected on the Diabetes Knowledge Test and the Diabetes Educational Profile.

\section{References}

1. Guidelines for diabetes care. New York and Pitman, New Jersey: American Diabetes Association and American Association of Diabetes Educators. 1981 .

2. Hess GE, Davis WK. The validation of a diabetes patient knowledge test. Diabetes Care 1983;6:591-96.

3. Davis WK, Hull A, Boutaugh ML. Factors affecting the educational diagnosis of diabetic patients. Diabetes Care 1981;4:275-78.

4. Hiss RG, Lengyel MC, Hess GE, Bowbeer LA, eds. Diabetes in communities. Ann Arbor: Michigan Diabetes Research and Training Center, 1986.

5. Beaser SB. Teaching the diabetic patient. Diabetes 1956:5:146-49.

6. Etzwiler DD. What the juvenile diabetic knows about his disease. Pediatrics 1962;29:135.

7. Etzwiler DD, Sines L. Juvenile diabetes and its managentent: family. social and academic implications. JAMA 1962;181:304.

8. Collier B. Etzwiler DD. Comparative study of diabetes knowledge among juvenile diabetics and their parents. Diabetes 1971;20:51-57. 